\title{
Ultrasonic Transceiver with the Possibilities of the Data Communication and the Two-Point Distance Measurement
}

\author{
Ondřej Lufinka \\ Faculty of Electrical Engineering \\ University of West Bohemia \\ Pilsen, Czech Republic \\ lufinkao@kae.zcu.cz
}

\begin{abstract}
This paper deals with the problem of the short distance measurement between two (or more) moving points with the ability of a simple data communication. Different possibilities were explored and using the ultrasonic transceiver was then chosen for a future development. The article describes a solution which uses ultrasonic transceivers and allows the distance measurement between two moving points (model masterslave) up to $5 \mathrm{~m}$ with the simultaneous communication (bit rate $100 \mathrm{bit} / \mathrm{s}$ ). The paper also proposes a possible future development to extend the model to more than 2 points distance measurement and communication.
\end{abstract}

Keywords-ultrasonic transceiver, distance measuring, data communication

\section{INTRODUCTION}

There are many methods available to measure a distance from a sensor to an obstacle. It is possible to use an infrared or an ultrasonic rangers, etc. This paper deals with the problem of the two moving points distance measurement where both of the points must be an active device capable of some communication and data processing to compute its position. Simultaneously it was required to implement a simple data communication between these devices.

In the beginning, different options were explored. The first idea was to use two GPS units that wirelessly communicate between each. The main disadvantage of this solution is a poor accuracy if one does not want to use really expensive devices. The next option is not to work with an absolute position but to measure just a relative position of the devices. It could be possible to do that with the help of Bluetooth or a similar wireless technology. This would be much cheaper, however the accuracy is not quite high either. So in the end it was chosen to explore a measurement through the modified proximity sensors. There are two options. Infrared or ultrasonic rangers. Infrared rangers have a smaller radiation angle, therefore ultrasonic devices are more suitable for this application. The ultrasonic distance measurement from a device to an obstacle is wellknown and was an inspiration for this article ([1], [2]).

There are different problems that need to be solved in order to develop a working prototype for this application and the following chapters are dealing with them. Theoretical principle, hardware and software development are described and the future possibilities for improvements are mentioned.

\section{THEORETICAL BACKGROUND}

The idea for the communication with the distance measurement is to create two points, each consisting of an ultrasonic transmitter and a receiver (Fig. 1). One of them is the master unit (initializes the communication), second one is the slave unit (responds to the communication). In the beginning, the master unit sends an ID with optional data that follow. If there is a slave with the same ID, it receives the communication and responds after the defined amount of time with its message. This message consists of the same ID and different data if needed. From the total amount of time for the communication it is then possible to compute the distance in the master unit. If we want to cover the whole space around the unit it must of course consist of more transmitter/receiver pairs. ID in the master unit can be changed and a different slave unit then responds. That enables communication to more slave units.

\section{A. Distance Measurement}

The distance calculation from the time of communication is shown in the Fig. 1 and then in the equations (1) and (2). The total time of communication consists of the time from master to slave $\left(t_{1}\right)$, the time for the slave unit to respond $\left(t_{2}\right)$ and the time from slave to master $\left(t_{3}\right)$. The distance can be easily calculated from $t_{1}$ and $t_{3}(1)$. But the master unit can measure only the total time $t$. So (2) is used for the calculation while the time $t_{2}$ is known (it is manually set in the slave unit).

Speed of the sound in the air is not constant. It is quite dependent on the temperature (approximately $0.18 \%$ per $1{ }^{\circ} \mathrm{C}$ ), therefore it is necessary to measure the temperature in the master unit as well. Dependency of the sound speed on the temperature is shown in (3), where $T$ is the temperature of the air in ${ }^{\circ} \mathrm{C}$.

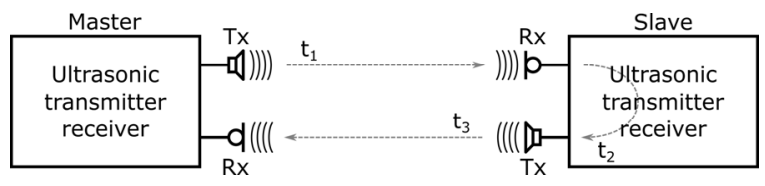

Figure 1. Communication between two points 


$$
\begin{gathered}
d=\frac{v_{\text {sound }} \times\left(t_{1}+t_{3}\right)}{2} \quad[\mathrm{~m}] \\
d=\frac{v_{\text {sound }} \times\left(\mathrm{t}-t_{2}\right)}{2} \quad[\mathrm{~m}] \\
v_{\text {sound }}=20,0457 \sqrt{273.15+T}[\mathrm{~m} / \mathrm{s}]
\end{gathered}
$$

The necessary precaution for the correct distance measurement is not to exceed the time for the slave to respond by the length of the message.

\section{B. Information Transmission}

With the idea described in the chapter II, it is already possible to send the data information while the distance is being measured at the same time. The next step for the successful information transmission is to develop a data coding (coding of the logical " 0 " and " 1 ", number of the pulses per bit and timing of the bits and words). This is shown in the Fig. 2.

Coding is resolved with the presence of pulses burst for the logical "1" in the bit window (with the period $T_{B}$ ) or the missing pulses for the logical " 0 ". The number of pulses $\left(N_{P}\right)$ must be big enough to successfully detect the transmission over the maximum distance (here $5 \mathrm{~m}$ ). In the ultrasonic transmission, both the amplitude and the length of the received pulses burst change with the distance. Amplitude decreases with the growing distance because of the signal attenuation. Length of the received pulses decreases with the distance because the transducer does not transmit/receive the signal with the square envelope. There are losses in amplitude in the beginning and at the end of the pulses burst because the transducer is capacitive and the smaller amplitude is not detected by the receiver with a higher distance. Therefore a coding with the amplitude or burst length is not possible. There is a space for improvement by choosing a different method such as the frequency modulation which on the other hand requires a wider band transducer.

The data word consists of the start bit (S) and given amount of the data bits (in this case there is a sequence of four data bits in the order from the start bit: "1", "1", " 0 " and " 1 "). The minimal period for the transmission of one data word $\left(T_{W}\right)$ can be calculated from the number of bits and the bit window period $\left(T_{B}\right)$. The bit period must be long enough so the burst from the previous bit with all its echoes does not affect the next bit. From the bit period the bit rate can be computed.

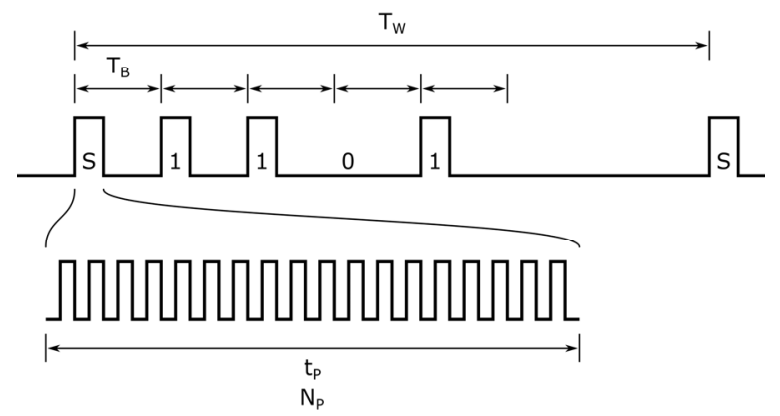

Figure 2. Coding and timing of the information
In the current concept the communication can go up to $100 \mathrm{bit} / \mathrm{s}$ with the bit period of $10 \mathrm{~ms}$.

\section{HARDWARE DEVELOPMENT}

The hardware solution is developed to allow one transducer as a combined transmitter/receiver. The driving of the transducer and the received signal processing can be done in any microcontroller. For the experiment the development kit FRDM-KE06Z was used. It was also used to power the different parts of the transceiver. Later a new board can be developed to be attached to the transceiver to replace the development kit. As was mentioned before, good idea would be to implement more transmitter/receiver pairs to one unit to cover the space around the unit. The current concept enables to communicate up to the distance of $5 \mathrm{~m}$.

\section{A. Transmitter Part}

In the transmitter, which is described in the Fig. 3, the key part is the transformer T1 with the voltage gain $1: 10$. It is used to drive the transducer REP1. With the square signal of the frequency of $40 \mathrm{kHz}$ on the port DRIVER, the primary winding of the transformer is being switched through the transistor Q1. The resistor R2 maintains the transistor closed when there is no DRIVER signal. The bipolar TVS diode D1 with the reverse voltage of $30 \mathrm{~V}$ protects the transistor against the voltage peaks caused by the inductance switching. The resistor R1 limits the maximal current through the primary winding of the transformer and the capacitor $\mathrm{C} 1$ covers the peak current consumption.

With the power supply voltage of $10 \mathrm{~V}$, there is a slightly deformed sine signal on the primary winding of the transformer, which creates a sine signal of the amplitude of $100 \mathrm{~V}$ on the secondary winding. This signal has an enough amplitude to drive the ultrasonic transducer. The second winding of the transformer (inductive character) with the transducer (capacitive character) together creates a resonant circuit. The resistor R3 makes a resistance load, which enables a discharge of the transformer, when the transistor is closed. The whole schematic is inspired from [2].

There are possible improvements in the transmitter part, especially in the optimization of the resonant circuit. That can lower the deformation of the sine signal, therefore the efficiency of the radiated power increases. The main advantage of this circuit is that the supply voltage can be quite low while the transducer is driven with a sufficient strength.

As mentioned before, there was a request to use one single transducer for a transmission and a reception as well. It can be seen in the schematic that the received signal can go through the resistor R4 and the capacitor $\mathrm{C} 2$ to the ECHO port, which is then connected to the receiver part (Fig. 4). The capacitor is good for the galvanic isolation of the receiver from the transmitter. A pair of the diodes D2A and D2B together with the resistor cuts off the voltage peaks higher than $1 \mathrm{~V}$ from the transmitter that could damage the receiver part. 


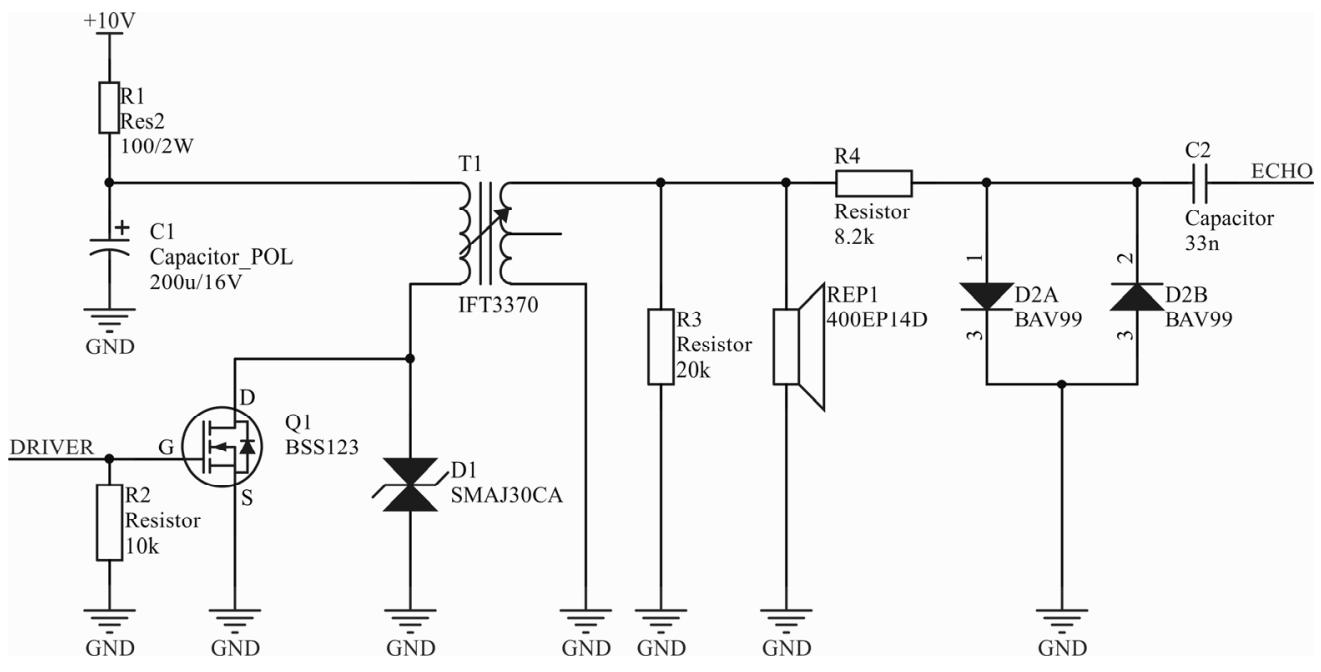

Figure 3. Schematic of the ultrasonic transmitter

\section{B. Receiver Part}

The receiver (Fig. 4) is designed as a double stage amplifier with a subsequent comparator with hysteresis. It was inspired by [3]. The amplifying part consists of two operational amplifiers (U2A and U2B) both connected in an inverting way. They belong to the same integrated circuit so only the part $\mathrm{A}$ has the power pins shown. The capacitors $\mathrm{C} 1, \mathrm{C} 3, \mathrm{C} 6$ and $\mathrm{C} 7$ do a galvanic isolation of the different stages. This also blocks the lower frequencies, which gives the whole amplifier a high-pass filter character. The capacitors $\mathrm{C} 2$ and $\mathrm{C} 5$ on the other hand limit the band from the high frequencies. This makes the amplifier behave as a low-pass filter. Together the circuit works as a band-pass filter with the center frequency of $40 \mathrm{kHz}$, which is also a resonant frequency of the transducer as well as the frequency of the transmission in this application. The resistors R3, R4, R6 and R7 set the total gain. The reference voltage of $2.5 \mathrm{~V}$ shifts the level of the signal in both of the amplifying stages to the center of the voltage range of the amplifiers, which is $0-5 \mathrm{~V}$. This enables to use the whole amplitude of the incoming signal.

The stage with two voltage dividers $(\mathrm{R} 8-\mathrm{R} 11)$ shifts the signal before the comparator module (U1) into two different levels. The signal on the non-inverting input of the comparator is shifted approximately to 427 $\mathrm{mV}$ and the signal on the inverting input to $371 \mathrm{mV}$.
The comparator has a hysteresis character with the limits of $391 \mathrm{mV}$ and $400 \mathrm{mV}$. Both the outputs are an open-drain type, which means any of them can pull the output low. The comparator behaves in the way, that if the non-inverting input falls lower than the down comparing limit, the output goes low. If the inverting input rises higher than the up comparing limit, it also pulls the output to the low level. Therefore if the signal from the amplifier stage has a small amplitude, the output is high ("1") because of the resistor R12. If the amplitude of the signal shifted for the comparator exceeds one of the comparing limits - the output goes active (low). So the receiver indicates the incoming signal of $40 \mathrm{kHz}$ pulses with the logical " 0 " pulses. This can be easily processed in the microcontroller which is connected to the port INT (interrupt). The gain of the amplifier and the values of the voltage dividers must be set in the way that the receiver detects the amplitude after the maximum transmission distance (here $5 \mathrm{~m}$ ). Also the noise must be taken into consideration. If the receiver was too sensitive, the output would behave randomly because of the input noise and also the noise coming through the supply voltage.

There is space to improve the receiver part mainly through creating a higher order band-pass filter, which enables better sensitivity and noise suppression of the receiver. There is also a possibility to use digital signal processing instead of the comparator.

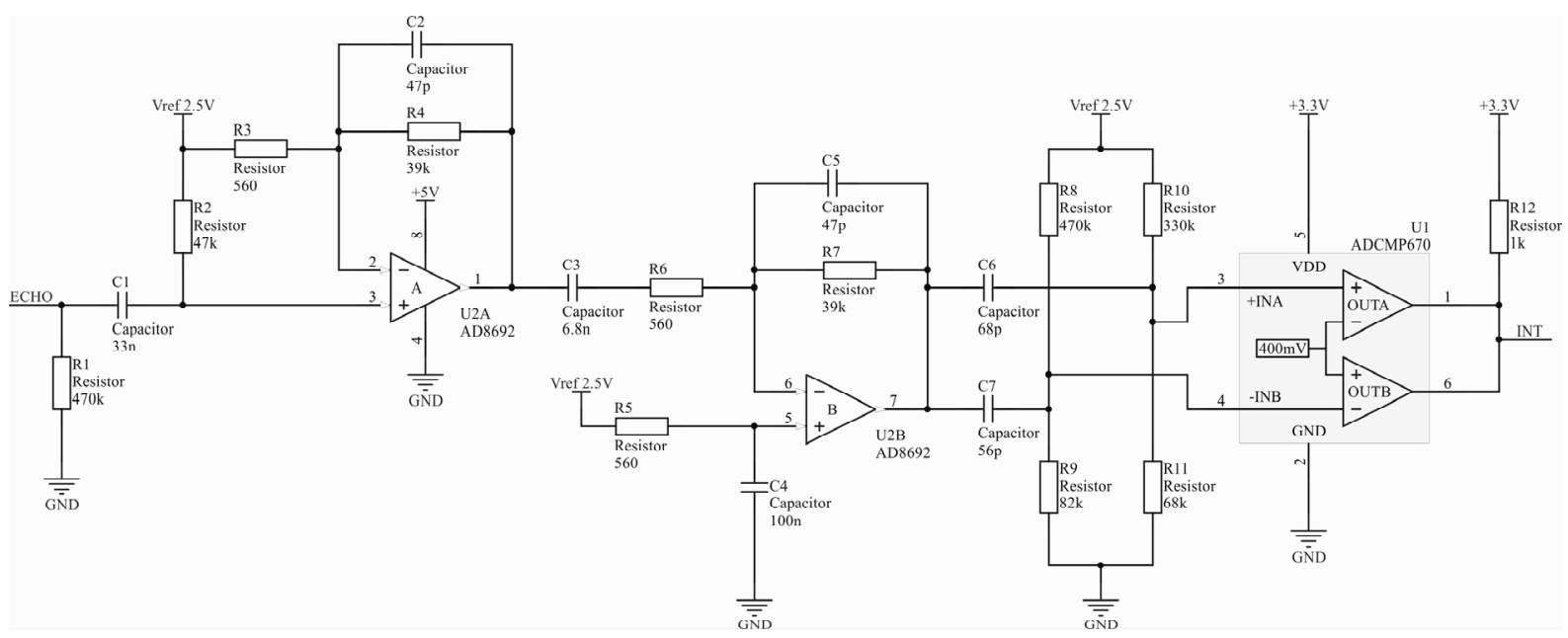

Figure 4. Schematic of the ultrasonic receiver 


\section{SOFTWARE SOLUTION}

The firmware to control the ultrasonic transceiver is designed for the FRDM-KE06Z development kit. There are two slightly different versions for the master and the slave unit. The main idea of the software solution is described in the Fig. 5.

The master unit takes care of initializing the transmission and asking for the response from the slave units. After the program starts, the unit waits for "Start to transmit" command, which can be for example an interrupt from a timer module. Then the receiving is disabled so its own transmission is not read as the incoming message. The message for the slave unit with the given ID and optional data is sent and the receiving is enabled again. The master unit then waits the defined amount of time for any slate unit to respond. If there is no response after the timeout, the slave unit with the current ID is marked as unreachable (it does not exist or the message was lost in one of the directions) and the algorithm is repeated.

In the meantime, all the slave units are listening for the messages from the master unit. When such a message arrives, it is received and decoded. Optional data can be used in the slave unit to perform some other task. If the ID is correct, the slave unit responds with its own message. Receiving of the slave unit is disabled during this transmission. Then the slave unit waits for the next message.

After that the master unit can receive the response (it means the slave unit with the current ID replied and the response was successfully delivered). If the message arrives, it is decoded. Data can be used again for any purpose. If the ID is correct the calculation of the distance is performed and the algorithm runs all over again.

This method can be used to communicate from one master unit to more slave units when the ID alternates before every transmission from the master unit. Interesting option would be to implement some more sophisticated protocol (e.g. CSMA-CA, CSMA-CD, etc.) where all the units could communicate between each other. Implementing CRC or self-correcting codes can be then the next step.

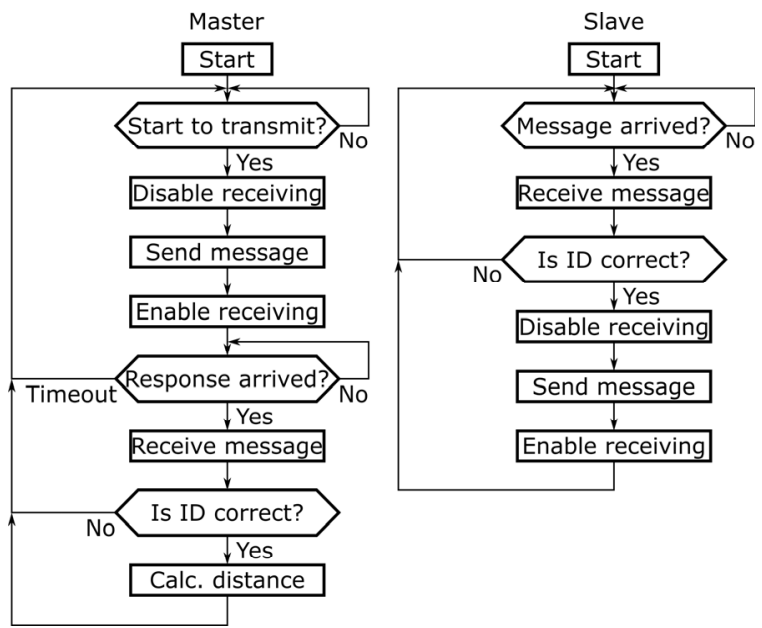

Figure 5. Software solution flowchart

\section{CONCLUSION}

The paper talks about the design of the ultrasonic system with the possibility of the data communication and the two point distance measurement. The concept enables communication between one master unit and more slave units. The master unit sequentially transmits requests with the given IDs to the slave units that send back their responses. From the total amount of time for the communication it is then possible to compute the distance in the master unit. Coding is resolved on the principle of a presence of pulses (" 1 ") or a non-presence of pulses (" 0 ") in the bit window. Hardware solution is designed in the way that one transducer can work for both transmitting and receiving. Firmware is developed for the FRDM-KE06Z board. The transceiver units are capable of communication over the maximum distance of $5 \mathrm{~m}$ with the bit rate up to $100 \mathrm{bit} / \mathrm{s}$.

So far this article shows an idea with the first working prototype and there are many possibilities for the future improvements that are already mentioned in the previous chapters as well. The concept can be improved by a different coding of the information (e.g. frequency modulation). The transmitter can be optimized to achieve a better radiated power by tuning the transducer resonant circuit. In the receiver part it is possible to implement higher order filters or replace the analogue detection (comparator) with a digital signal processing. Also the measurement to explore an error rate in the communication and an accuracy of the measured distance will be part of the next work.

This kind of the communication/distance measurement can be used in the applications where a relative position of more moving points in the small distances is important and using an absolute position (GPS) is not sufficiently precise or is too expensive (differential GPS). For example it could work to synchronize more drones or autonomous vehicles in the combination of a height measurement and a distance measurement from a reference point without an external observation system.

\section{ACKNOWLEDGMENT}

This project has been supported by the Ministry of Education, Youth and Sports of the Czech Republic under the Research Project SGS-2015-002, Faculty of Electrical Engineering, University of West Bohemia.

\section{REFERENCES}

[1] S. Zhang, "Research of Ultrasonic Distance Measurement Device," 2010 6th International Conference on Wireless Communications Networking and Mobile Computing (WiCOM), Chengdu, 2010, pp. 1-3.

[2] H. He and J. Liu, "The Design of Ultrasonic Distance Measurement System Based on S3C2410," Intelligent Computation Technology and Automation (ICICTA), 2008 International Conference on, Hunan, 2008, pp. 44-47.

[3] Pro-Wave Electronics Corporation, "Sonar ranging modules SRM400" [online], (C) 2005, Pro-Wave Electronic Corp., last change October 2013 [cit. 2016-03-20], available: http://www.prowave.com.tw/english/item/download.htm

[4] Analog Devices, "Ultrasonic distance measurement" [online], (C) 2014-2015, Analog Devices, Inc., last change 2015-08 [cit. 2016-03-20], available: http://www.analog.com/en/designcenter/reference-designs/hardware-reference-design/circuitsfrom-the-lab/cn0343.html\#rd-overview 